\title{
Four Subjects in Solar Physics from the Point of View of the Electric Current Approach
}

By Syun-Ichi Akasofu

University of Alaska

Abstract- Four major subjects in solar physics, the heating of the corona, the cause of the solar wind, the formation of sunspots and the cause of solar flares, are discussed on the basis of the electric current approach, a sequence of processes consisting of power supply(dynamo), transmission (currents/circuits) and dissipation(high coronal temperature, solar wind, sunspots and solar flares). This is because the four subjects have hardly been considered in terms of the electric current approach in the past, in spite of the fact that these subjects are various manifestations of electromagnetic processes. It is shown that this approach provides a new systematic way of considering each subject; (1) the long-standing issue of the coronal temperature, (2) the long-standing problem on the cause of the solar wind, (3)the presence of single spots (forgotten or dismissed in the past) and its relation to unipolar magnetic regions and (4) the crucial power/energy source and subsequent explosive processes of solar flares. The four subjects are obviously extremely complicated and difficult subjects, but it is hoped that the electric current approach might provide a new insight in considering the four subjects.

Keywords: coronal temperature solar wind sunspots solar flares.

GJSFR-A Classification: FOR Code: 020109

Strictly as per the compliance and regulations of:

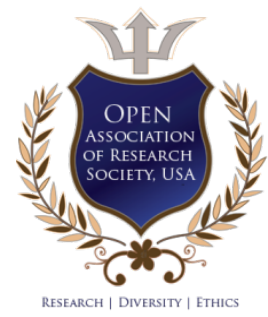

(c) 2021. Syun-Ichi Akasofu. This research/review article is distributed under the terms of the Attribution-NonCommercialNoDerivatives 4.0 International (CC BY-NC-ND 4.0). You must give appropriate credit to authors and reference this article if parts of the article are reproduced in any manner. Applicable licensing terms are at https://creativecommons.org/ licenses/by-nc$\mathrm{nd} / 4.0 \%$. 


\title{
Four Subjects in Solar Physics from the Point of View of the Electric Current Approach
}

\author{
Syun-Ichi Akasofu
}

Abstract- Four major subjects in solar physics, the heating of the corona, the cause of the solar wind, the formation of sunspots and the cause of solar flares, are discussed on the basis of the electric current approach, a sequence of processes consisting of power supply(dynamo), transmission (currents/circuits) and dissipation(high coronal temperature, solar wind, sunspots and solar flares). This is because the four subjects have hardly been considered in terms of the electric current approach in the past, in spite of the fact that these subjects are various manifestations of electromagnetic processes. It is shown that this approach provides a new systematic way of considering each subject; (1) the longstanding issue of the coronal temperature, (2) the longstanding problem on the cause of the solar wind, (3)the presence of single spots(forgotten or dismissed in the past) and its relation to unipolar magnetic regions and (4) the crucial power/energy source and subsequent explosive processes of solar flares. The four subjects are obviously extremely complicated and difficult subjects, but it is hoped that the electric current approach might provide a new insight in considering the four subjects.

Keywords: coronal temperature solar wind sunspots solar flares.

\section{InTRODUCTION}

In solar physics, there are at least four major issues.

1. Heating of the corona to more than $10^{6} \mathrm{~K}$,

2. Cause of the solar wind,

3. Process of the sunspot formation,

4. Cause of solar flares.

Each theory/observation was initiated by:

1. Observed high temperature of the corona (cf. Van de Hulst, 1953).

2. Theory of the solar wind (Parker, 1958).

3. Theory of the formation of sunspots (Babcock, 1961).

4. Theory of magnetic reconnection (Sweet, 1958).

Thus, the first two issues have been lasting at least a half-century. At the present time, there is no promising theory in both problems. The last two have widely-accepted theories, but there are serious contradictions (the presence of single spots, for example) and uncertainties (the process of energy source), respectively.

Author: International Arctic Research Center, University of Alaska, Fairbanks, Alaska, USA. e-mail: sakasofu@alaska.edu
Each of the above issue has respectively been studied by the following principle and premise.

1. The corona is heated by MHD waves from the photosphere.

2. The cause of the solar wind is based on the heated corona.

3. A pair of sunspots is caused by a rising magnetic flux tube.

4. Solar flares are caused by magnetic reconnection.

The present situation in each has been reviewed and summarized. Some of the recent reviews are:

1. All efforts of heating by MHD waves and others do not seem to succeed (cf. Van Doorsselaere et al., 2020).

2. All efforts of generating the solar wind do not seem to work (cf. Viall and Borovsky (2020).

3. The problem of the presence of single (unipolar) spots and the location of pairs of spots with respect to unipolar magnetic regions are not considered (Akasofu, 2021).

4. The theory of magnetic reconnection has been presumed to be the source of energy production process and has almost exclusively prevailed in the past. A number of simulation studies have been conducted. However, after the most extensive review based on multi-satellite observations and others, Fletcher et al. (2011) are uncertain about the role of magnetic reconnection as the energy source process.

On the other hand, in as early as 1967, under the title "The second approach in cosmical electrodynamics", Alfven (1967) emphasized the need for electric current approach in space plasma physics, as well as the magnetic field line approach. The electric current approach considers solar phenomena systematically as a sequence of processes, which consists of power supply (dynamo), transmission (currents/circuits) and dissipation (solar phenomena). He repeated this point later (Alfven, 1977, 1981,1986). By neglecting the particle and electric current aspects, he warned: "we deprive ourselves of the possibility of understanding some of the most important phenomena in cosmic plasma physics."

The four subjects are extremely complex and difficult. However, in spite of a great progress in 
observations, the above long standstill and/or stagnation in theoretical considerations on them seem to stem from what Alfven pointed out in the above.

In fact, there is one common weakness or lack in studying all the four subjects. It is the lack of considering them on the basis the electric current approach, which consists of a sequence of processes, power supply (dynamo), transmission (currents/circuits) and dissipation (observed phenomena).In fact, this sequence of processes is the basic way of studying electromagnetic phenomena. Many aspects of the four phenomena cannot quantitatively be explained without electric currents and electron flows.

Therefore, in this paper, the electric current approach is adopted in studying the four major subjects. It seems that this approach seems to provide a new way of considering them.

\section{il. High Temperature of the Corona}

The standard coronal temperature of $2 \times 10^{6} \mathrm{~K}$, corresponds to $170 \mathrm{ev}$, based on the ionization potential of highly ionized Fe ions. It seems to be difficult to find common plasma or wave processes from the photosphere to heat the corona to $10^{6} \mathrm{~K}$.
However, the ionization by energetic electrons is another possibility for the presence of highly ionized atoms and the high temperature of the corona (Stix, 2002), but the responsible process of the acceleration of electrons in the coronal environment has not been explored.

It is suggested that one ionization current system is a field-aligned current system along coronal magnetic loops, which consists of a photospherc dynamo process as the power supply for the fieldaligned currents, because field-aligned currents are essential in developing a double layer, which is needed to accelerate both electrons and ions.

\section{a) Power supply/Dynamo and Circuit/currents}

The dynamo process $(V \times B)$ is caused by a plasma flow across a magnetic field. The power of a dynamo is defined by the Poynting flux $P$ (erg/s or w) bot phenomena is given by:

$$
P=\int(E \times B) \bullet d S=V\left(B^{2} / 8 \pi\right) S,
$$

where $V$ and $B$ dente the velocity of photospheric plasma flow and the magnetic field, respectively and $S$ is the cross-section of the dynamo process (Figure 1a).

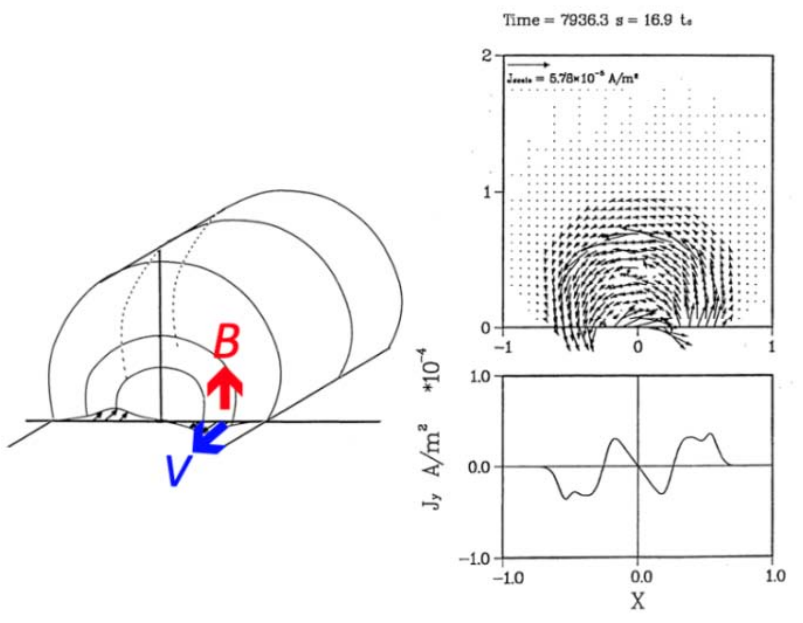

(a)

(b)

Figure 1: (a) The dynamo process in the photosphere for solar flares under a magnetic arcade. (b) The dynamo induced current system. The field-aligned currents occur along the magnetic arcade field lines(Choe and Lee, 1998; Courtesy of G. S. Choe).

Typical values of the parameters are:

Photospheric plasma speed $V=2.0 \mathrm{~km} / \mathrm{s}$,

Magnetic field intensity $=B=100 \mathrm{G}$,

$\mathrm{S}$ (cross-section) $=\mathrm{k} \times \mathrm{d}, \mathrm{k}=$ lateral dimension $(1.0 \times$ $\left.10^{5} \mathrm{~km}\right), \mathrm{d}=$ the depth $\left(6.0 \times 10^{4} \mathrm{~km}\right)$,

$P=2.4 \times 10^{27} \mathrm{erg} / \mathrm{s}$.
This dynamo system is considered again in Section 6, because a similar dynamo power can supply the power for solar flares.

An example of the dynamo-induces fieldaligned currents under a magnetic arcade is shown in Figure $1 \mathrm{~b}$, in which the dynamo process is considered with a set of $B=6 \mathrm{G}$ and the speed $V$ of $2 \mathrm{~km} / \mathrm{s}$ along the neutral line under a typical magnetic arcade (cf. Choe and Lee, 1996); the intensity of field-aligned current is about $5 \times 10^{-5} \mathrm{~A} / \mathrm{m}^{2}\left(5 \times 10 \mu \mathrm{A} / \mathrm{m}^{2}\right)$. 


\section{b) Current/circuits: Double layer}

In the past, a double layer was once considered for solar flares and its effect on coronal processes (Li et al., 2013, 2014). However, no estimate of the ionization rate by a double layer has made for a given current intensity and its possible potential drop along magnetic field lines. The presence of an electric field associated with the double layer along magnetic field lines for auroral processes was suggested by Alfven (cf. 1981, 1986) on the ground that magnetospheric electrons have to be accelerated to penetrate into the upper atmosphere and that the magnetospheric electric current system has to close itself (current continuity) by penetrating into the ionosphere at an altitude of $110 \mathrm{~km}$ from the magnetosphere.

The presence of the double layer (a U-shaped potential structure) in field-aligned currents is observationally well confirmed by several satellite observations in auroral research. The aurora is simply a result of this visible process.

In the earth's auroral conditions, various observed values related to the double layer are summarized by Karlsson (2012) and others: Fieldaligned potential drops of the order of $6 \mathrm{KV}$ or more, field-aligned currents of $10^{-1}-10^{1} \mu \mathrm{A} / \mathrm{m}^{2}$, and the acceleration of magnetospheric electrons from $300 \mathrm{eV}$ to $10 \mathrm{KeV}$ and more, an estimated thickness of the double layer $10 \mathrm{KV}$ per $1 \mathrm{~km}$, located between 0.5-2.0 $\mathrm{Re}$ above the ionosphere ( $\mathrm{Re}=$ the earth's radius).

In the following, we estimate the ionization in the corona.

\section{c) Dissipation (Ionization)}

Neutral hydrogen atoms area major constituent in the middle height of the corona Aschwanden, 2005, his figure 1.19). The equation for the ionization rate $q$ by a beam of energetic electrons in the ionosphere is given by (cf. Rees 1989):

$$
q=F E \rho \mathrm{d} / \mathrm{R} E^{2} \times 30 \mathrm{ev} / \mathrm{cm}^{3} \mathrm{~s} .
$$

The ionization rate of the middle level of the coronaby field-aligned currents of $0.001 \mathrm{~A} / \mathrm{cm}^{2}$ $\left(10^{3} \mathrm{~mA} / \mathrm{cm}^{2}\right)$, supposing that the double layer provides of potential drop $1 \mathrm{KV}$ :

$F=$ electron flux $\left(6.2 \times 10^{11} / \mathrm{cm}^{2}\right)$,

$E=$ electron energy $(1 \mathrm{Kev})$,

$\rho=$ mass density $\left(1.6 \times 10^{-13} \mathrm{~g}=10^{11} / \mathrm{cm}^{3} \times 1.6 \times 10^{-24} \mathrm{~g}\right)$,

$d=$ length $\left(10^{9} \mathrm{~cm}\right)$,

$\mathrm{RE}^{2}=$ effective range $\left(5.6 \mathrm{~g} / \mathrm{cm}^{2}\right)$,

$q=6.3 \times 10^{8} / \mathrm{cm}^{3} \mathrm{~s}$.

Thus, it seems that the proposed field-aligned currents along magnetic loops can ionize hydrogen atoms and $\mathrm{Fe}$ atoms in the middle level of the corona.

\section{ili. Cause of the Solar Wind}

Since all the attempts to produce the solar wind in the past have so far been based on the coronal heating or small-scale electromagnetic processes in the corona, it is very difficult to overcome the solar gravitational force. Thus, it may be worthwhile to consider other causes of the solar wind.

The process must be a large-scale bodily electromotive force (the Lorentz force, $J \times B$ ) in order to lift the whole heliospheric plasma.

a) Solar unipolar dynamo

We suggest that the solar unipolar induction and the associated electric currents playan important role in generating the solar wind. The solar unipolar induction was considered by Alfven(1950, Chapter 3, p.5; 1977, 1981). However, the importance of the unipolar induction on the generation of the solar wind has not specifically been considered in the past.

\section{b) Currents/circuit}

The currents and circuits of the solar unipolar dynamo is shown in Figure 2a. In Alfven's model, the electric current flows out from the northern pole of the sun along the polar axis. After reaching the pole of the heliosphere, the current flows along the assumed spherical surface of the heliosphere to the equatorial plane (longitudinal current) and then flows back radially to the sun on the magnetic equator. On the equatorial plane, there is also the thin circular current, which is known as the equatorial current sheet. Both the radial current and the circular current on the equatorial current together generate spiral currents, which generate Parker's spiral field lines. The magnetic field produced by the longitudinal current is shown in Figure $2 \mathrm{~b}$ (Akasofu et al., 1980).Alfven (1981) estimated the total current intensity to be $1.5 \times 10^{9} \mathrm{~A}$.

The resulting electromotive force $(J \times B)$ must satisfy:

(1) The $(J \times B)$ force must have a significant outward component.

(2) The velocity of the solar wind is fairly uniform as a function of latitude during the solar minimum period. 


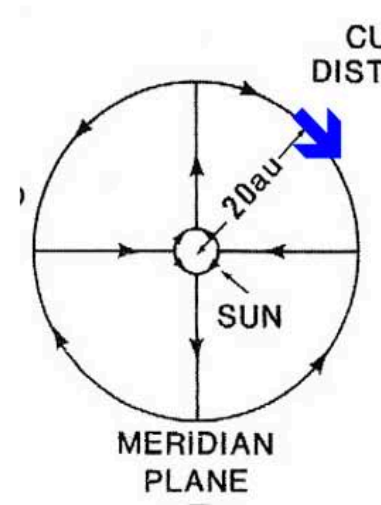

CURRENT DISTRIBUTION

\section{c) Dis}

Dissipation: Generation of the solar wind

Although the proposed $(J \times B)$ force is much greater than the solar gravitational force, it is difficult to lift the whole heliospheric plasma, if the longitudinal current is located only at the outer surface of the heliosphere or distributed uniformly in the heliosphere.

Lee and Akasofu (2021) considered that one third of the current flow on the spherical shell between $r=9.5-10.5 R_{\odot}$ around the sun, not all from the top of the heliosphere; thus, in this shell, it is assumed that current $\mathrm{I}=5 \times 10^{8} \mathrm{~A}$ (current density $\mathrm{j}=2.3 \times 10^{-11} \mathrm{~A} / \mathrm{m}^{2}$ ), magnetic field $B=1.0 \times 10^{3} \mathrm{nT}, \mathrm{N}=100 / \mathrm{cm}^{3}\left(10^{8} / \mathrm{m}^{3}\right)$. The resulting acceleration $a=j B=1.4 \times 10^{2} \mathrm{~m} / \mathrm{s}^{2}$, compared with the solar gravity at 10 solar radii = $2.8 \mathrm{~m} / \mathrm{s}^{2}$. The time for the solar wind with $500 \mathrm{~km} / \mathrm{s}$ to move one solar radius $T=1.4 \times 10^{3} \mathrm{~s}$. Thus, the solar wind thus produced at $1 \mathrm{au}$ is $V=a T=200 \mathrm{~km} / \mathrm{s}$ under the solar gravitation force.

Thus, it may be necessary to consider a large-scale $(J \times B)$ force for a cause of the solar wind

\section{Formation of Sunspots}

Babcock (1961) proposed that sunspots appear as a positive and negative pair, when a thin magnetic flux tube below the photospheric surface emerges by magnetic buoyancy at the respective cross-sections, as shown in Figure $3 \mathrm{a}$ and $3 \mathrm{~b}$. Since then, the concept of the rising thin magnetic flux tube has prevailed until the present.

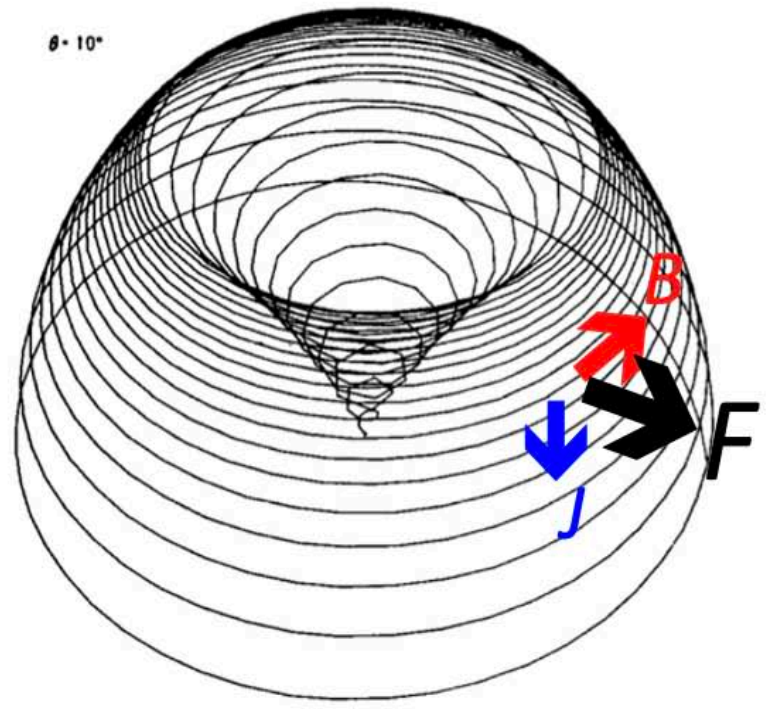

(b)
There are several serious morphological difficulties of this thin magnetic flux tube theory. They are:

(1) There are many single spots, often called isolated, independent, solitary spots or unipolar spots (the present theory indicates that spots should appear always as a (positive and negative) pair.

(2) Unipolar magnetic regions grow and decay with the sunspot cycle(what have been thought to be old active regions), so that they are one of the basic features of solar magnetism related to the sunspot cycle.

(3) Positive spots are formed in a positive unipolar region (vice versa) as a local coalescence of pores.

(4) A pair of clusters of spots (positive and negative) are formed only at the boundary of neighboring (positive and negative) unipolar regions, constituting the magnetic pair connection (the magnetic buoyancy suggests the occurrence of pairs at any location).

(5) It is difficult explain the Butterfly diagram (the pair can occur any place). These are difficult to explain by Babcock's theory.

Therefore, an entirely new morphological study may be needed on the formation of sunspots. A new morphology can be considered by synthesizing these observed facts.

In Figure 3c, as one of the basic facts, it may be noted that a single spot consists of several pores, not a single column of magnetic flux tube. The size of a single spot depends on the number and size of pores. 


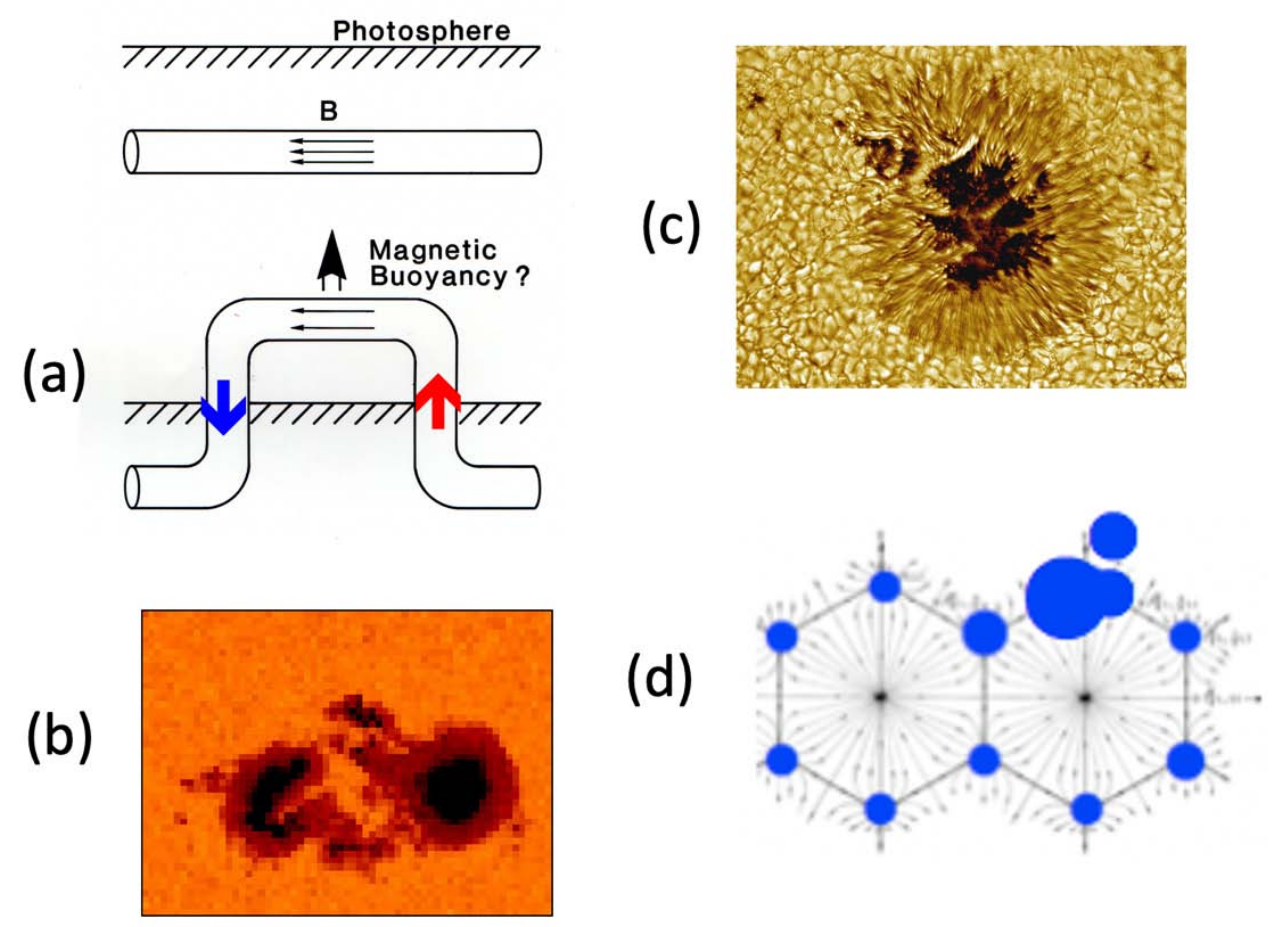

Figure 3: $(a, b)$ The presently accepted theory of the formation of sunspots, in which a pair of spots (positive and negative) appears when an assumed magnetic flux tube rises from below the photospheric surface. (c) An example of single spots (NASA sunspot collection). (d) Photospheric convection cells with pores and single spots (assembly of pores); for the convection cells, see Clark and Johnson (1967).

This subsection deals with a new morphology of sunspots (Akasofu, 2021), before considering theoretical implications.

\section{a) A new morphology}

The first important clue in understanding the single (unipolar) spot was the presence of positive and negative unipolar magnetic regions (Akasofu, 2015). Figure 4a shows the distribution of magnetic fields on the solar disk. One can recognize that unipolar regions are weak positive and negative bands, aligned alternately in longitude, stretching well above the latitude of sunspots; there are also the northern and southern Polar Unipolar Regions. After examining the Kitt Peak solar magnetic maps during the three sunspot cycles $(21,22,23)$, the first new findings are: (1)Unipolar regions are found to grow and decay with the sunspot cycle (Figure 5). (2) Unipolar regions are sometimes connected to the Polar Unipolar Regions. (3) Unipolar regions appear before sunspots at the beginning of a new sunspot cycle (Akasofu, 2021). Therefore, unipolar magnetic regions should be considered as one of the basic feathers of solar magnetism related to the sunspot cycle. In the past, they have been considered to be decaying active sunspot groups, which spread by the non-uniform rotation of the sun (Leighton, 1969). 
Figure 4: (a) A low resolution image of the distribution of magnetic fields on the solar disk. (b) A schematic illustration of the magnetic distribution on the solar disk, unipolar magnetic fields, concentrated fields (single spots, cluster of single spots (marked by a dot with circle and spots, which may be considered to be a pair of spots) in the network configuration. (c) A small section of the boundary of two unipolar regions with pores, single spots and clusters of spots forming a pair.(d) An example of pairs of spots; the left side is an assembly of small spots and right side perhaps two large spots (coalescence of single spots) and a few single spots (NASA Sunspot collection).

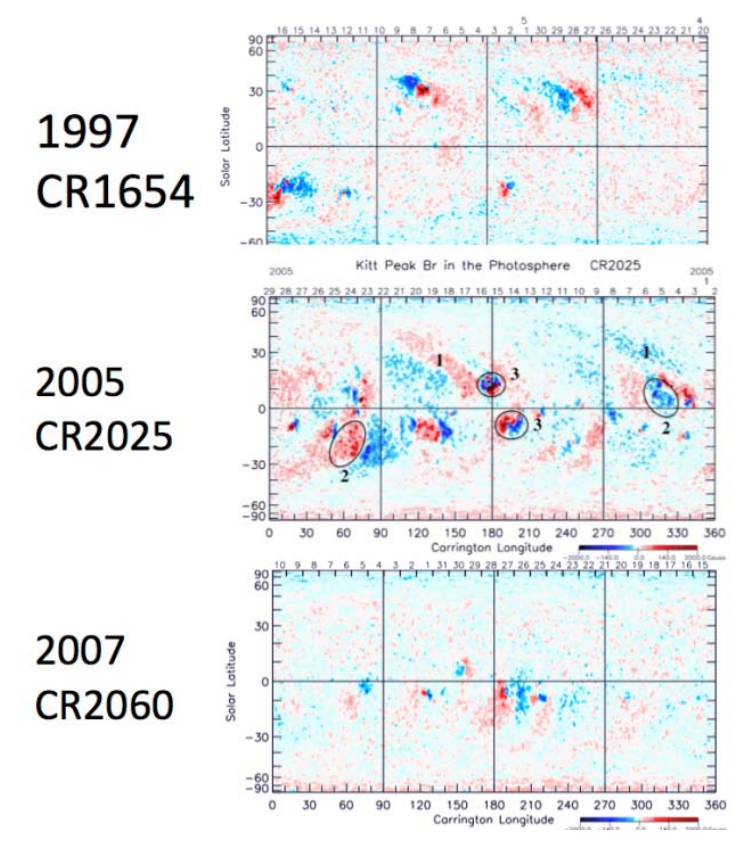

Figure 5: The distribution of magnetic fields on the photosphere, from the top, an early, middle and end epochs of the sunspot cycle, showing that unipolar regions grow and decay with the sunspot cycle. 
In Figure 4a, there are also concentrated fields in unipolar regions; they are pores, single spots and clusters of spots.

The second important clue in Figure $4 \mathrm{a}$ is the fact that positive single spots (red dots) are formed in a positive unipolar region (red region) and vice versa; This feature is schematically shown in Figure $4 \mathrm{~b}$, which shows a summary of a study of the relationship between unipolar regions and single spots during three sunspot cycles (cf. Akasofu, 2015).

Figure $4 \mathrm{c}$ is a schematic enlarged view of a small portion of Figure $4 \mathrm{a}$ and $4 \mathrm{~b}$ near the boundary of two unipolar regions, showing pores (dots), single spots (several dots together) and clusters of spots, forming a pair. Figure $4 \mathrm{~d}$ shows an example of pair of clusters, constituting a pair across the boundary (a cluster consists of single spots).

\section{b) Dynamo process/currents: Formation of single spots}

The above morphological study requires a new way of considering the formation of sunspots, although a large number of studies based on the rising thin magnetic flux tube have been made in the past (cf. Solanki, 2003; Rempel, 2011).

We consider first the fact that the convective motions are always present in the photosphere. The convection cells are known to accumulate some magnetic fields along their boundaries (namely, local concentrations along the boundaries) because of irregularities of the convective motions, thus forming a network of pores, which appears as a uniform field from a global point (low resolution) of view (Figure 4a).

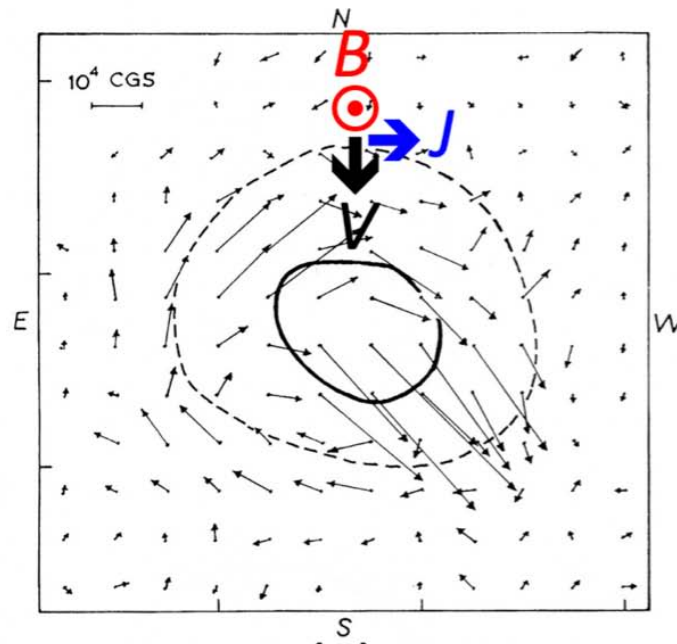

(a)
It is also known that these irregularities "coalesce" (not merging into single), forming pores, and then pores coalesce to form a single spot at some focal points of the boundary of the cells (Mclntosh, 1981);this situation is schematically shown in Figures $3 d$ and 5c.Parker (1992) considered vortex attraction for the coalescence. Thus, a spot is not a simple column of magnetic flux.

Based on the new facts in (a), it is proposed that the formation of single spots is a local coalescence and conversion of unipolar magnetic fields, namely positive ones in a positive unipolar region (vice versa).

Plasma motions are important in the formation of sunspots, not just the magnetic buoyancy in the thin tube theory. The local concentration can happen when a local convergence occurs; a powerful converging and downward flow down to the depth of $1.0 \mathrm{Mm}$ was observed (cf. Zhao et al., 2001).

In a magnetic field, the local plasma convergence is associated with electromotive force $(V \times B)$ and resulting current $J$. Kotov (1971) showed that there is a circular electric current around a small single spot of about $10^{12} \mathrm{~A}$; Figure 6a. In fact, even Babcock's thin magnetic flux needs also a thin solenoidal current, which requires a plasma flow toward the central line of the tube and the resulting $(V \times B)$ process.

In a large-scale view, considering the formation of a smallest spot $\left(B=500 \mathrm{G}\right.$, the radius $1.5 \times 10^{3} \mathrm{~km}$; Allen, 1955), the size of the converging area will be about 7 times of the area of a small spot by assuming the intensity of a unipolar region of 10G; this size corresponds to the size of large spots.

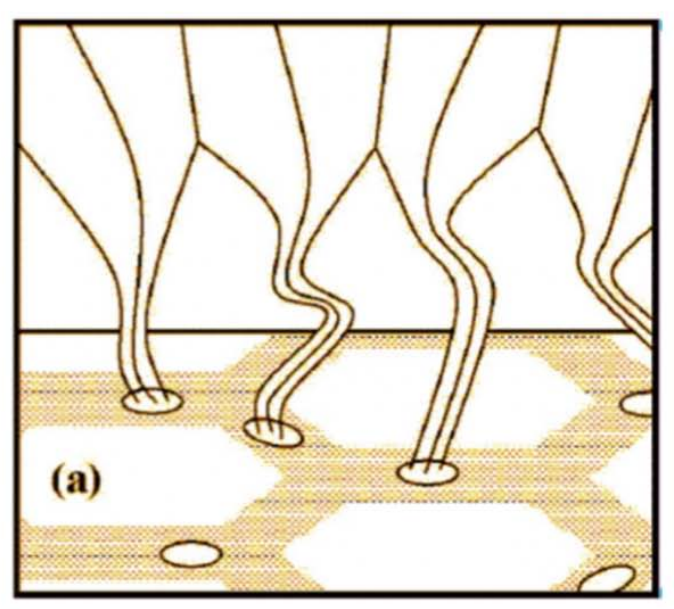

(b)

Figure 6: (a) Electric current around a single spot (Kotov,1971) with the electromotive force $(\mathrm{VX} B)$ and the current vector $\mathrm{J}$. (b) The magnetic field above pores/single spots in the magnetic field network (taken from Canmer, 2009 for a different context)

The above process of a local photospheric convergence as the process of coalescence is expected to cause little change in the upper part of a unipolar region, and thus no magnetic flux adjustment with neighboring unipolar regions is needed; Figure $6 \mathrm{~b}$. This is perhaps only way to overcome the problems of 
unipolar magnetic spots; in the past, it has generally been assumed that single spots must have their counterpart somewhere, but without considering concretely the location.

Further, in the past, the formation of spots has hardly been discussed in terms of dynamo process and the associated electric currents. In considering a converging motion of plasmas for the dynamo process, it is important to know that there is also an outflow from the top of spots is well known as the Evershed flow (Bray and Loughhead, 1964; Solanki,2003).

\section{c) Formation of pairs of spots}

One of the most important new findings in the formation of pairs of spots in the above morphological study is that a pair of clusters of spots is located only at the boundary of neighboring (positive and negative) boundary of unipolar regions. They do not form in the middle of a unipolar region; Figures $4 \mathrm{~b}$ and $\mathrm{c}$; the thin tube theory considers only magnetic buoyancy, so that pairs of spots could appear in any place on the solar disk.

Figure 4c shows schematically a pair of two clusters, forming pairs of spots at the boundary of neighboring unipolar regions, in addition to the magnetic network of unipolar regions (positive and negative), pores, and single spots. Large spots (500$2000 \mathrm{G}$ ) are also known to be the coalescence of many single spots (Mclntosh,1981).

Figure $4 d$ shows an example of two clusters (pairs of spots) in each side of neighboring unipolar regions. The main feature in the left side shows a large number of single spots, while in the right side a large spot (actually, three coalesced single spots); the two clusters form a pair. An important point is that two clusters of spots across the boundary are not symmetric at all, so that it is unlikely that they are inherently connected as a tube under the photospheric surface; this is generally the case for the neighboring two clusters at the boundary.
In this view, single spots are the basic unit of sunspots, not a pair of spots.

Since pairs of spots form at the boundary of neighboring unipolar regions, it is expected that the magnetic connection between two active groups of single and large spots occurs in a way, by which Sheeley (1976) presented; on the basis of X-ray images of magnetic field lines, he described: "---these field lines usually interact by changing their flux linkage, much as they do in a vacuum". That is to say, for a given magnetic field distribution of two clusters at the boundary of neighboring unipolar regions, the magnetic connection between them may occur almost like in a potential field case.

The basic difference between the tube theory and the present morphological theory is that the present theory is developed on the basis of the observed magnetic records, while the tube theory is based on an assumed magnetic flux tube, which has so far not been detected yet.

\section{d) Butterfly diagram}

Any theory of the formation of sunspots must explain the equator ward shift of spots as the solar cycle advances, namely the Butterfly diagram. Since we are dealing with a new morphological theory (not the assumed equator ward winding of the thin magnetic flux tube), it is necessary to find a related observed fact. Howard and La Bonte (1980) found a large-scale latitudinal tortional oscillation, which shifts equator ward during each solar cycle; Figure 7. Mclntosh (1981) showed that there occur sunspots and flare activities along the oscillation belt. Thus, there must be a close relationship between the boundaries of unipolar regions and the torsional oscillation belt. Note again Parker's vortex theory on the coalescence.

If sunspots were caused by a thin magnetic flux tube (thin solenoidal current) by magnetic buoyancy, sunspots would appear at any place and any time on the solar disk.

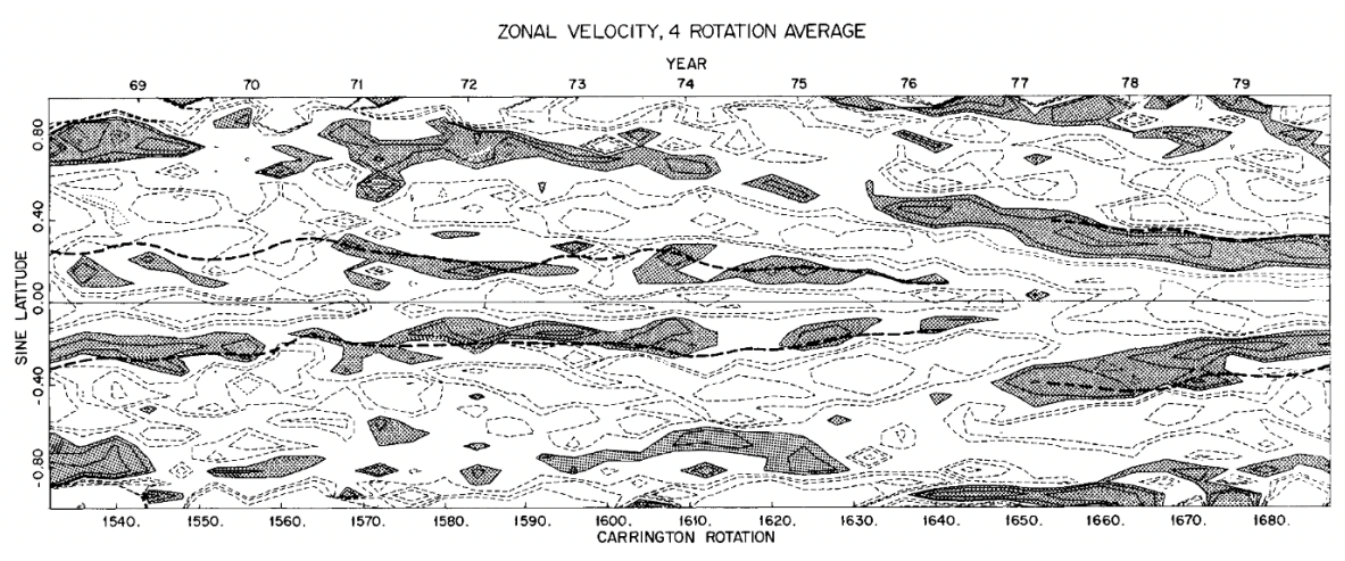

Figure 7: A large-scale tortional oscillation of the sun, which shits equator ward during each sunspot cycle (Howard and La Bonte, 1980). 


\section{e) Dissipation}

When one observes a later stage of an active sunspot group, it appears as a scattered pores and single spots. Thus, the first stage of dissipation is a dispersing process of coalesced pores and single spots (see Bray and Loughhead, 1964; the $\beta \gamma$ type in p.234) by the non-uniform rotation of the sun, rather than disintegration of a single magnetic flux tube. It is most likely that the ohmic loss will eventually dissipate individual scattered single spots.

The thin magnetic tube theory has several difficulties,(1) the presence of single spots; (2) the close relationship between single spots with unipolar magnetic regions; (3) the location of pairs of spots with the boundary of neighboring unipolar regions and (4) the Butterfly diagram. Thus, the formation of spots should be based on a new morphological theory of the formation of sunspots.

A study of sunspots is still an early phase of morphological study, so that a theoretical study here is very incomplete. As the first basic process of the formation of sunspot formation is a local convergence of unipolar regions, associated with electromotive force $(V \times B)$ and current $J$ in the photosphere, forming pores and single spots. Pair of spots are formed only at the boundary of two unipolar regions, interacting with the large-scale torsional oscillations.

A single spot is the basic unit of spots, not a pair of spots.

\section{Solar Flares}

An explosive process depends generally on accumulated energy, which can be suddenly released. The theory of magnetic reconnection presumes that magnetic reconnection processes has such characteristics. Thus, unfortunately, magnetic reconnection has been presumed to be an explosive process for more than one-half century. Thus, the past efforts are concentrated in find plasma processes which could have the desired reconnection rate (explosive) under an anti-parallel magnetic configuration, but after several decades, there is so far no clear agreement on the rate and the produced amount of energy. Thus, there is even a possibility that magnetic reconnection is a result of flare processes, not the cause.

It may be recalled that after analyzing the reconnection theory, Parker (1963) concluded: "The observational and theoretical difficulties with hypothesis of magnetic field annihilation [magnetic reconnection] suggest that other alternatives for the flare must be explored."

Petschek (1964) appeared to be able to respond to Parker's criticism. However, after a most recent extensive review of observations, Fletcher et al. (2011), recalling Parker's criticism, mentioned that they are not certain about whether magnetic reconnection is the energy release process. Thus, we are back to 1963. Fletcher et al. (2011) noted also that because of the presumption, the supporting observations of the simultaneous occurrence of flares have the problem of confusing the cause-effect relationship. Thus, it is uncertain if magnetic reconnection is really the cause of solar flares. Further, there has been not much effort how the generated fast flow of plasma by magnetic reconnection can explain various flare phenomena. The $\mathrm{H} \alpha$ emission requires the knowledge of electric currents.

Another obvious possibility of an explosive energy source is to consider a loop current, which can accumulate needed magnetic energy (Alfven, 1950, 1981), but its possibility as the energy source was forgotten or not considered.

Here, we consider solar flares as a result of the sequence of processes, which begin with a dynamo process as the power supply and a current loop is the source of energy.

\section{a) Dynamo and current/circuit}

The basic mode of the dynamo of solar flares is an arcade-mode dynamo discussed Section 2. Figure $8 a$ is the same as Figure 1a.

Plasma flows along the neutral line is about 1.6 $\mathrm{km} / \mathrm{s}$ (Young et al., 2004) or much higher (cf. Min and Chae, 2002). 

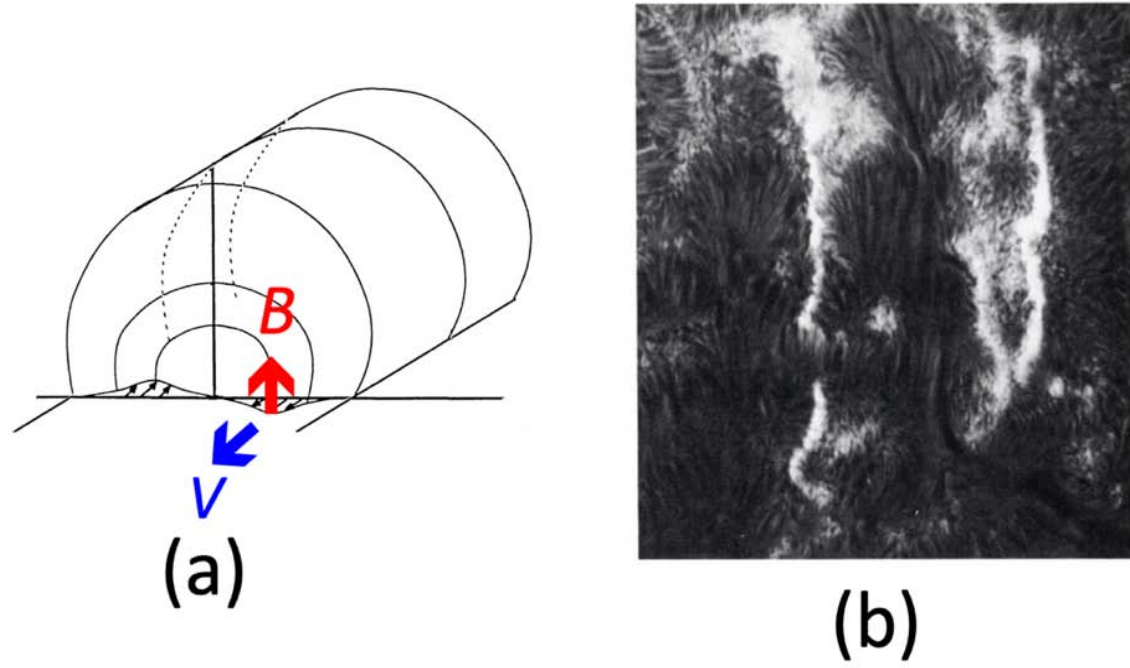

Figure 8: (a) The photospheric dynamo process under amagnetic arcade. (b) An example of spotless flares (Svestka, 1976).

\section{b) Dissipation: Spotless flares}

The dynamo under the arcade produces tworibbon flares at the feet of the arcade, because the fieldaligned currents along the arcade can excite chromospheric atoms. These flares are called spotless flares, because there is no sunspot around them. Actually, they occur more frequently than ordinary flares.

Although spotless flares are generally weak, they are the basic feature of flares driven by a dynamo process under the electric current approach. An active sunspot group provides regions of a stronger $B$ field (see the power equation in Section 2), not sunspots themselves. The reason why intense flares tend to occur in an active sunspot group is due to this fact.

A significant part of the energy dissipation is associated with the $\mathrm{H \alpha}$ emission resulting from the enhancement of the field-aligned currents along the arcade magnetic field lines; it is about $10^{31}$ erg (Svestka,1976).Very energetic phenomena associated with flares, such as solar sub-cosmic rays, dissipate less energy in terms of the total energy budget.

\section{c) Explosive process: The other dissipation}

In addition to the magnetic field-aligned currents along the magnetic arcade, the dynamo process in the magnetic arcade model produces a loop current along the two-ribbon emission (Akasofu and Lee, 2019). It flows along the dark filament between the two ribbons, but above them (Figures9a and b). 


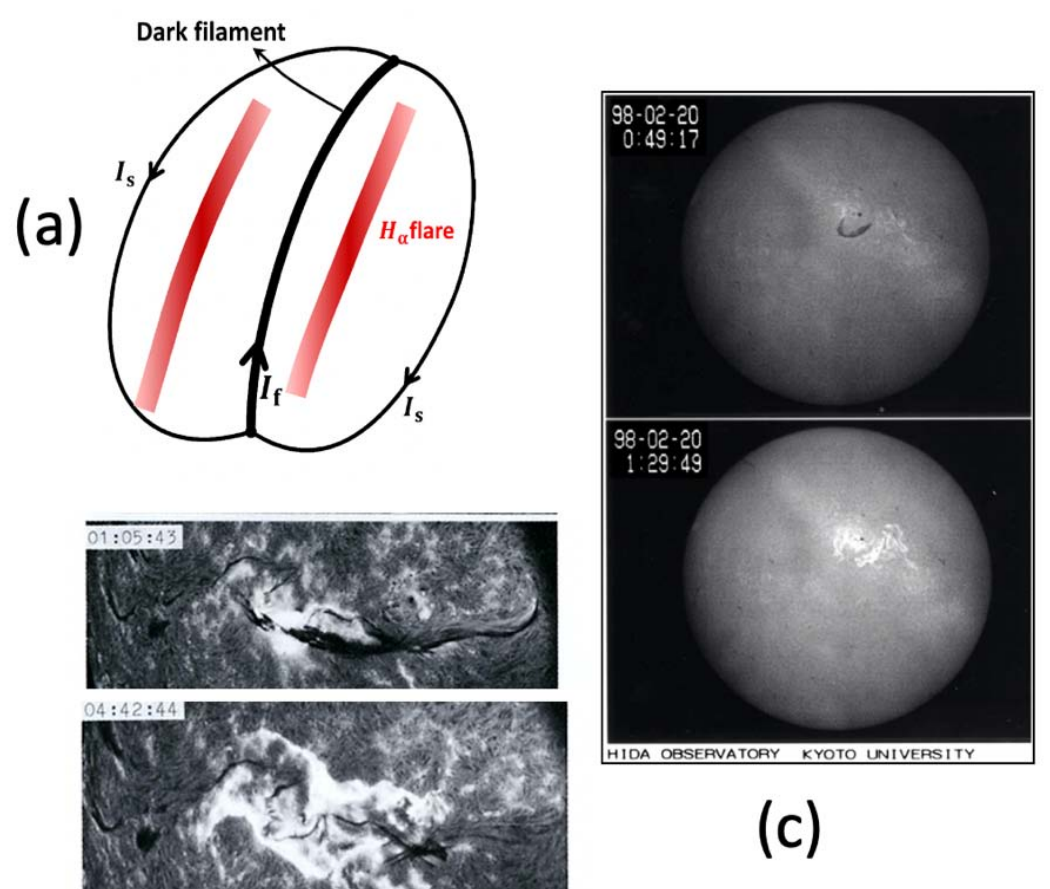

(b)

Figure 9: (a) The arcade dynamo generates another current between the two-ribbon flare, but above it. (b) Upper: an image just before flare onset; note the dark filament. Lower: the maximum epoch of a two-ribbon flare; note that the dark filament was blown away (Courtesy of E.Hiei, Norikura Solar Observatory). (c) An example of dispartion brusques (DB; Courtesy of E.Hiei, Norikura Solar Observatory)

The loop current has magnetic energy of $W=$ $(1 / 2) I^{2} L$, where I and $L$ denote the current intensity and inductance, respectively. For a typical vale $\mathrm{I}=10^{11} \mathrm{~A}$ (Chen and Krall, 2003) and $L=2000 \mathrm{H}$ (Alfven, 1981), $W=10^{32} \mathrm{erg}$, so that the loop current along the dark filament can have enough energy for flares.

One of the ways to observe the energy accumulation is to examine magnetic shear (Wang et al., 1994); in his case, the shear increased for about 5 hours before flare onset, so that the power in this case is estimated to be $2.8 \times 10^{26} \mathrm{erg} / \mathrm{s}$ (Akasofu and Lee, 2019); the speed of plasma estimated in this study is in agreement with the flow speed, $1.6 \mathrm{~km} / \mathrm{s}$, observed by Yang et al. (2004).

The reasons for emphasizing that the exploding loop current as the energy source of the explosive phase of solar flares is that the disappearance of the dark filament occurs at the time when the two-ribbon emission is greatly enhanced, flare onset (Figure 9b and c). This phenomenon is described in detail by Svestka (1967, p.229) as "dispartions brusques, (DB)"; DBs seem to occur in association with explosive feature and are likely to be caused by a current instability in the loop current. Indeed, Kurokawa et al. (1987) showed that the exploding prominence (the filament) has unwinding motions, indicating a reduction of the electric currents along the loop.
It is unfortunate that DBs are hardly considered today (perhaps, the attention has been focused only on magnetic reconnection).

Therefore, a magnetic arcade dynamo generates field-aligned currents along the arcade magnetic field lines, producing a two-ribbon flare and a loop current along the dark filament. The loop current in the dark filament has enough magnetic energy for flares. Therefore, the photospheric dynamo is likely to the power source of flares, and thus the loop current along the dark filament is another or a better source of the explosive energy than magnetic reconnection.

\section{Vi. Concluding Remarks}

In this paper, the following suggestions are made for each of the four subjects on the basis of the electric current approach.

(1) Coronal heating: The photosphericdynamo and coronal magnetic loop currents for the ionization of the corona by energetic electrons.

(2) Solar wind: Solar unipolar induction, the associated circuit and currents for the electromotive force $(J \times B)$ as adriving current system for the solar wind.

(3) Sunspot formation: The dynamo process of the photospheric convergence flow and associated currents are crucial. 
(4) Solar flares: The photospheric dynamo under a magnetic arcade, and the resulting the field-aligned current along the arcade field lines produce the tworibbon emission. The dynamo generates also the current loop along the filament for the explosive process.

It is hoped that these suggestions are useful in pursuing the four subjects and that the electric current approach will be considered in the future.

\section{ACKNowledgements}

The author would like thank Hannes Alfven who emphasized that I should take the electric current approach, when we met first time in 1966 and many times later. The author would like to thank L.-C. Lee for extensive discussions on solar physics.

\section{References Références Referencias}

1. Akasofu, S.-I. (2015). Single spots, unipolar regions and pairs of spots, Geophys. Res. Lett., 42, Doi: 1002/2014GL062887.

2. Akasofu, S.-I. (2021). A morphological study of unipolar regions and the relationship with sunspots, J. Atmosph and Solar-Terr. Physics, 218, (2021) 105625. https//doi.Org/10.1016/j.jastp.2021.105625.

3. Akasofu, S.-I., P.C. Gray, and L.C. Lee, A model of the heliospheric magnetic field configuration, Planet. Space Sci., 28, 609-615, 1980.

4. Akasofu, S.-I. and L.-C. Lee, 2019, On the explosive nature of auroral substorms and solar flares: The electric current approach, J. Atmosph. SolarTerr.Phys., 186 (2019), 104-115.

5. Alfven, H., 1967, The secondapproach to cosmicalelectrodynamics, in The Birkeland Symposium on Aurora and MageticStorms, ed. By A. Egeland, J. Holtet, Centre National de la Rescherche, Paris, 439-444.

6. Alfven, H. 1977, Electric currents in cosmic plasmas, Rev. Geophys. Sapce Phys., 15, 271-284.

7. Alfven, H., 1981, Cosmic Plasma, D. Reidel Pub. Co.Dordrecht-Holland

8. Alfven, H. 1986. Double layers and circuits in astrophysics, 1986, IEEE trans. On Plasma Phys.,PS-14, No.6.

9. Aschwanden, M., 2005. Physics of the Solar Corona, Springer, in association with Praxis Pub., Chichester, UK.

10. Babcock, H. W. (1961) The topology of the sun's magnetic field and the 22-year cycle, Astophy.J., 133, 572-587.

11. Bray, R. J. and Loughheard, R. E. (1964). Sunspots, Dover.

12. Chen, J. and J. Krall, 2003, Acceleration of coronal mass election, J. Geophys. Res., 108, SSH-2, doi. 10. 102903JAA009849.
13. Choe, G.S. and L. C. Lee, 1996a, Evolution solar magnetic arches I. Ideal MHD evolution under footpoint shearing, Astrophy. J., 472, 360-388.

14. Clark, A. and Johnson, A. C. (1967). Magnetic field accumulation in supergranules, Sol. Phy.,2, 433440.

15. Cranmer S. R. (2009, Coronal holes, Living Rev. Solar Phys., 6, 3 https://www.livingrviews.org//rsp2009-3.

16. Fletcher, L., B. R. Dennis, H. S. Hadson, S. Krucker, K. Phollips, V. Veronig, M. Batterglia, L. Bone, A. Caspi, Q. Chen, P. Gallagher, P. T. Grigis, H. Ji, W. Liu, R. Q. Milligan and M. Temmer, 2011, An observational review of solar flares, Space Sci. Rev. (2011) 159: 19-106, doi: 10. 1007/s1214-010-97018.

17. Howard, R. and B. J. La Bonte, The sun is observed to be a tortional oscillator with a period of 11 years, Astrophys. J., 239, L33-L36.

18. Kurokawa, H. Y., K. Hanaoka, K. Shibata and Y. Uchida, 1987, Rotating eruption of untwisting filament triggered by the $3 \mathrm{~B}$ flare of 25 April, Solar Phys., 108, 251.

19. Karlsson, T., 2012. The acceleration region of stable auroral arcs. Auroral Phenomenology and Magnetospheric Processes: Earth and Other Planets, 227-239. Ed by A. Keiling, E. Donovan, F. Bagenal and T. Karlsson, Geophys. Monograph Series 197, AGU, Washington, DC.

20. Kotov, V. A. (1971). 213-219, IAU Symposium No.43, held at the College de France, August 31September 4, 1970.ed by Howard, R., D. Reidel Pub. Co., Dordrecht-Holland. Clark, A. and Johnson, A. C., 1967, Magnetic field accumulation in supergranules, Solar Phys., 2, 433-440.

21. Lee, L.-. C. and Akaofu, S.-I. 2021. On the causes of the solar wind: Part 1. Unipolar solar induction currents, J. Geophys. Space Phys., 126, 1-8, e2021JA029358. https://doi.org/10.1029/2021JA 029358.

22. Li, T. C., Drake, J. F. and Swisdak, M., 2013, Coronal electron confinement by double layers, Astrophys. J., 778 (2). Art. No.144.

23. Li, T. C., Drake, J. F. and Swisdak, M., 2014, Dynamics of double layers, ion acceleration, and heat flux suppression During solar flares, Astrophys. J. 793 (1). Art. No.7.

24. Leighton, R. B. (1969). A magneto-kinematic model of the solar cycle,Astrophys. J., 156, 1-26.

25. McComas, D. J., Angold, N., Eliott, H.A., Livadiotis, G., N.A. Schwadron, N. A., Skoug, R. M. and Smith, C. W. (2013). Weakest solar wind of 779:2 (10pp), December 10.

26. Mclntosh, P.S. (1981). The Physics of Sunspots, Vol.7, ed by Crom, L.E. and Thomas, J.H., pp. 7-54, Sacramento Peark Observatory, New Mexico. 
27. Min, S. and J. Chae, 2009, The rotating sunspot in AR 10930, Solar Phys., (2009), 258:203-217, doi: 10. 1007/s11207-009-9425-7.

28. Parker, E. N. (1958). Dynamics of theinterplanetary gas andmagnetic field, Astrophys, J., 128, 664-676, https://doi.org/10.1086/146579.

29. Parker, E. N. (1992). Vortex attraction and formation of sunspots, Astrophys. J.,390, 290-296.

30. Petschek, H. E., 1964, Magnetic field annihilation, in the Physics of Solar Flares, Proceedings of the AASNASA Symposium held 28-30 October 1963, at the Goddard Space Flight Center, Geeenbelt, MD., ed. by W. H. Hess, Wahington, DC: NASA, Science and Technical Information Division.

31. Rempel, 2011, Penumral fine structure and driving mechanisms of large-scale flows in simulated sunspots, Astrophy. J.,729-5 (22p)

32. Rees, M. (1989). Physics and Chemistry of the Upper Atmssphere, Cambrige Universiy Press.

33. Sheeley, N. R. Jr. (1976) Energy released by the interaction of coronal magnetic fields, Solar Phys., 47, 177-185.

34. Solanki,S. K., 2003, Sunspots: Anoverview, Astron. Astrophys. Rev., 11,153-286. Doi: 10.1007/s00159003-18-4

35. Sheeley, N. R. Jr. (1976) Energy released by the interaction of coronal magnetic fields, Solar Phys., 47, 177-185.

36. Stix, M., 2002. The Sun, Springer-Verlag, Berlin Heidelberg.

37. Svestka, Z., 1976, Solar flares, D. Reidel Pub. Co. Dordrecht $=$ Holland.

38. Sweet, P. A.,1958, The neutral point theory of solar flares, 123-134, in Electromagnetic phenomena Cosmical Physics, ed. by B. Lehnert, Cambridge University Press.

39. Van Doorsselaere, T., Srivasttava, A. K., Antolin, P., Magyare, N., Farahanni, S. V. et al. (2020.) Coronal heating by MHD waves. Space Sci. Rev., 2020, 216140, https://doi.org./10.1007/s1124-020

40. Van de Hulst, H. C., 1953. The chromosphere and the corona. p.207-321, in The Sun, ed. By G. P. Kuiper, Univ. Chicago Press.

41. Vasyliunas, V. M., 1975, Theoretical models of magnetic fiel line merging, Rev. Geophys., Space Phys., 13,303-336.

42. Viall, N. M. and Brovsky, J. E. (2020). Nine ousstanding questions of solar wind physics, J. Geophys. Res., Space Physics, 125, e2018JA0 26005, https://doi.org/ 10.1029/2018JA026005.

43. Wang, H., Ewell, M. W.and Zirin, H. !1994). Vector magnetic field changes associated with X-class flares. Astrophys. J., 424,436-443

44. Young, G., Y. Xu, W. Cao, H. Wang, C. Denker and T. R. Rimmele, 2004, Photospheric shear flows along the magnetic neutral line of active region 10486, Asrophys. J. 617: L151-154.
45. Zhao, W. W., A. G. Kosovichev and T. L. Duvall, Jr., 2001, Investigation of mass flows beneath a sunspot by time-distance helioseismology, Astrophys. J., 557:384-388,

46. Zirin, H., 1988. Astrophysics of the Sun, Cambridge University Press, Cambridge.

47. Verscharen, D., Kristopher, K. G. Klein and Maruca, B. A., The multi-scale nature of the solar wind, Living Rev. in Solar Phys.(2019) 165 https://doi.1007/s 1116-018-0021-0. 